\title{
Perceptual selectivity in immature and adult rats
}

\author{
WILLIAM A. ROBERTS
}

VASSAR COLLEGE

\begin{abstract}
Immature and adult rats under both high and low drive conditions were trained to discriminate between two compound stimuli differing along the dimensions of direction, thickness and form. By introducing test trials which varied only one dimension at a time, it was found that animals organized the problem solely in terms of the direction dimension and did not attend to thickness or form.
\end{abstract}

\section{Problem}

In a previous paper (Roberts, 1965), some experiments were reported which investigated the possibility of qualitative differences in learning between immature and adult rats. No evidence of qualitative differences was found, immature rats showing early progressive improvement in habit-reversal and maximizing in probability-learning just as had been found in adult animals. In the experiment reported here, the search for qualitative differences is extended to the area of perceptual organization. Immature and adult rats were trained to discriminate between two compound stimuli, differing along three dimensions. When criterion was reached, the discrimination was maintained while test trials were randomly introduced on which stimuli were presented which held two dimensions equivalent and varied the third. Selection of any dimension was indicated on test trials by above chance responding to the correct component of that dimension. An ontogenetic comparison was made by comparing the patterns of results found with immature and adult animals.

In addition, drive state was varied at each age level to control for the possibility that perceptual organization is influenced by level of motivation. That drive may be an important factor is suggested by some experiments of Bruner, Matter, \& Papanek (1955) in which it was found that high drive levels reduced the number of cues which adult rats learned about.

\section{Method}

The Ss were 12 immature and 12 adult male and female albino rats of the Wistar strain. At each age level, animals were divided into a high drive and a low drive group, each containing six Ss. Immature animals were put on a feeding schedule whereby food was available for $6 \mathrm{hr}$. each day, but the High Drive Group was trained 15-17 hr. deprived and the Low Drive Group was trained 1-3 hr. deprived. With adult animals, the High Drive Group was maintained at $80 \%$ of ad lib feeding weight and the Low Drive Group was maintained at $95 \%$ of ad lib feeding weight. They were kept on a $24 \mathrm{hr}$. feeding schedule, fed hard pellets $1 / 2 \mathrm{hr}$. after each day's training session.
The apparatus consisted of two gray wooden modified Lashley jumping stands, one for adult animals and a scaled down model for young animals. Each stand was made up of an elevated Y-platform with a stem which branched into two runways, each of which led to a window in the face of the apparatus. Behind the windows was the rectangular goal compartment. Both apparatus sat on legs which raised the Y-platform 40 in off the floor, and both stands had tops and sides which projected outward from the face at an angle forming a funnel which served to eliminate extraneous stimulation.

Preliminary training was identical for immature and adult animals; each $\mathrm{S}$ was trained to run down the runways, push over mid-gray cards in the windows of the stand, and enter the goal compartment. All animals were rewarded on each run with wet mash which they were allowed to nibble for a few seconds from a dish placed at the back of the goal compartment.

At the beginning of experimental training, the immature animals were 27 days old and the adult animals were 97 days old. The animals were trained to discriminate between pairs of stimulus cards, 5 in by 4.5 in for adults and 4 in by 3.5 in for the immature animals, which presented compound stimuli differing along three dimensions, direction, thickness and form. Each compound was made up of a gray circle or upright triangle form on a ground of black and white thick or thin horizontal or vertical stripes. The thick stripes were .5 in wide and the thin stripes were .25 in wide. The forms used in the adult stimulus compounds were an equilateral triangle with a 2.12 in base and 2.25 in sides and a circle with a 2.12 in diameter while the forms for immature animals were an equilateral triangle with a 1.62 in base and 1.88 in sides and a circle with a 1.62 in diameter. On all cards, the forms were placed .75 in above the bottom edge, centered with reference to the sides of the card.

There were eight possible stimulus compounds and four possible pairs of compounds to be discriminated. In each group, individual Ss were assigned to stimulus pairs so that each component was in a correct compound an equal number of times. There were 20 training trials each day, the correct compound being on the right and left an equal number of times according to Gellermann orders. A modified correction procedure was used. If $S$ nosed the incorrect card, which was locked in place, it was brought back to the stem of the $\mathrm{Y}$ and given an opportunity to correct the error; if $S$ made three repetitive errors on a trial, it was guided to the correct stimulus on the next run. Discrimination training was 
carried to a criterion of $90 \%$ or better correct responses on each of two consecutive days.

When an animal reached criterion, it was given 10 days of testing on which test trials were randomly interspersed among original discrimination trials. On test days, the original training compounds were presented on 14 trials, the incorrect stimulus locked in place. On the six test trials, three in the first 10 trials and three in the second 10, pairs of compounds, excluding the training compounds, were presented which held two dimensions equivalent and varied the third. Each dimension was varied once in the first three trials and once in the second three trials, the correct component for each dimension being on the right for one trial and on the left for the other trial. Neither of the stimulus cards presented on test trials was locked in place, a response to either one being rewarded. Over the 10 test days, there were 20 test trials on each dimension for each animal. Selection of any dimension was determined by significantly greater than $50 \%$ response to the correct component on test trials.

\section{Hesults}

During the course of the experiment, one animal in the Immature-Low Drive Group was discarded because of refusal to perform, and the results for that group are based on an $\mathrm{N}$ of 5 . Table 1 presents a groups by dimensions analysis of the test trial choices with the data broken down into the first five test days and the last five test days. The significance of positive deviations from $50 \%$ was tested by t-tests, and it can be seen that on test days 1-5, all four groups significantly selected the correct component of the direction dimension while they responded at chance on both the thickness and form dimensions. On test days $6-10$, all groups again

Table 1. Mean Percentage of Responses made to the Correct Component on Test Trials

\begin{tabular}{|c|c|c|c|c|c|c|}
\hline \multirow[b]{2}{*}{ Group } & \multicolumn{3}{|c|}{ Days $1-5$} & \multicolumn{3}{|c|}{ Days $6-10$} \\
\hline & 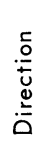 & $\begin{array}{c}n \\
n \\
0 \\
\frac{x}{u} \\
\frac{u}{v} \\
\stackrel{E}{E}\end{array}$ & $\begin{array}{c}E \\
\text { ㅎํ } \\
\text { ए }\end{array}$ & 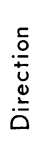 & $\begin{array}{l}n \\
u n \\
0 \\
\frac{0}{0} \\
\frac{u}{E} \\
E\end{array}$ & $\begin{array}{l}E \\
\text { 호 }\end{array}$ \\
\hline Immature-Low Drive & $82^{\star}$ & 54 & 46 & $78^{*}$ & $60^{\star}$ & 50 \\
\hline Immature-High Drive & $88^{*}$ & 58 & 53 & $82^{\star}$ & 55 & 48 \\
\hline Adult-Low Drive & $98^{*}$ & 43 & 47 & $90^{*}$ & 57 & 60 \\
\hline Adult-High Drive & $95^{\star}$ & 45 & 53 & $88^{\star}$ & $62^{\star}$ & 55 \\
\hline
\end{tabular}

* Significantly greater than $50 \%$ at the .05 level of confidence. responded significantly above chance on the direction dimension and at chance on the form circe jion. On the thickness dimension, however, two groups, Immature-Low Drive and Adult-High Drive, chose the correct component significantly greater than $50 \%$ of the trials.

\section{Diseussion}

These results are clearly of a noncontinuity variety; apparently the animals organized the original discrimination solely in terms of vertical and horizontal stripes and learned nothing about stripe thickness or form. That two groups responded significantly above chance to the correct thickness component on the second block of test days suggests that on test days 1-5 some animals learned to attend to the thickness dimension through inspection of test stimuli which differed only in thickness, and, through attention to the thickness dimension on non-test discrimination trials, came to learn about the correct and incorrect thickness components.

There is no suggestion of an ontogenetic difference in perceptual organization in these data, the pattern of results for immature animals being identical to that for adult animals. Furthermore, drive level was not a significant variable at either age level. These conclusions, of course, must be restricted to the stimuli used. For instance, in a recent experiment of very similar design to this one, Sutherland \& Mackintosh (1964) trained adult rats to discriminate between compounds containing direction and brightness cues. On test trials, animals showed selection of both dimensions, responding to the correct brightness component $72 \%$ of trials and the correct direction component $86 \%$ of trials. The difference in the Sutherland and Mackintosh results and the results of this experiment suggests that the number of dimensions selected or attended to in a discrimination between compound stimuli will depend on the nature of the dimensions involved. Further experiments with immature and adult animals using compounds which vary along a variety of dimensions may yet reveal ontogenetic changes in perceptual organization.

\section{References}

Bruner, J. S., Matter, J., \& Papanek, M. L. Breadth of learning as a function of drive level and mechanization. Psychol. Rev., $1955,62,1-10$.

Roberts, W. A. Learning and motivation in the immature rat. Amer. J. Psychol., 1965, in press.

Sutherland, N. S., \& Mackintosh, J. Discrimination learning: Non-additivity of cues. Nature, 1964, 201, 528-530. 